\title{
A COLETA DE SÊMEN POST MORTEM PARA REPRODUÇÃO HUMANA ASSISTIDA E OS DIREITOS DA CRIANÇA
}

\author{
Post mortem semen collection for human assisted reproduction \\ and children's rights
}

\begin{abstract}
Luiz Antonio da Costa Sardinha', Maria Valéria de Omena Athayde', Simey de Lima Lopes Rodrigues', Marcia Raquel Panunto Dias Cunha', Venâncio Pereira Dantas Filho', Helder José Lessa Zambelli', Yliah Cavalcanti Sardinha², Derli Conceição Munhoz ${ }^{4}$, Ilka de Fátima Ferreira Boin ${ }^{3}$
\end{abstract}

\section{RESUMO}

Os autores descrevem dois casos de solicitação, pelas esposas, de coleta de sêmen post mortem, em pacientes com diagnóstico de morte encefálica, para posterior utilização em reprodução humana assistida. Diante do inusitado da situação, foi realizada avaliação ética e legal dos personagens envolvidos no processo, com especial atenção à criança a ser gerada, sua situação legal e linha sucessória e a melhor postura ética a ser tomada pelo profissional médico envolvido no processo.

Descritores: Morte Encefálica; Doação de Órgãos; Sêmen; Inseminação Artificial; Células Germinativas; Família.

\footnotetext{
Instituição:

1 Organização de Procura de Órgãos do Hospital de Clinicas da Universidade Estadual de Campinas - Campinas/São Paulo, Brasil.

2 Advogada, autônoma, formada pela UNESP - Franca

3 Departamento de Cirurgia da Faculdade de Medicina da Universidade Estadual de Campinas - Campinas/SP - Brasil

4 Departamento de Anestesia - Hospital das Clínicas Unicamp, Campinas/SP, Brasil

Correspondência:

Luiz Antonio da Costa Sardinha

End.: Hospital de Clinicas da Unicamp - Campus de Barão Geraldo Campinas/SP

Tel.: (19) 3521-8000

E-mail: captacao@hc.unicamp.br
}

\section{INTRODUÇÃO}

A solicitação de coleta de sêmen post mortem não é inédita na literatura. Nos anos 1980, Rothman 1 descreveu a primeira solicitação e coleta de sêmen em paciente em morte encefálica (ME) e doador múltiplo de órgãos, dando ênfase à parte técnica da realização desse procedimento e levantando aspectos éticos em sua realização. Nesse mesmo período, o mundo vivenciou alterações importantes nos modelos de constituição da família e os incríveis avanços na tecnologia, no processo de reprodução humana assistida, criando nova expectativa para as mulheres e para a sociedade. 
A concepção de uma criança de pai falecido tornouse possível após a implementação da tecnologia de criopreservação, e, na maior parte dos casos, o pai havia deixado consentimento expressando seu desejo. ${ }^{2}$

Alguns autores descrevem sua preocupação com relação à educação da criança de um pai falecido e do antigo modelo de famílias com somente um dos pais, ${ }^{3-5}$ fato hoje que não constitui novidade.

O processo de doação de órgãos e tecidos em pacientes com morte encefálica (ME) é regulamentado e legalizado na maioria dos países, inclusive no Brasil, por meio da Lei $9434 / 97^{6}$ e da Resolução $1480 / 97 .^{7}$

Durante o referido processo, além das dificuldades impostas pelo tema, os médicos vêm deparando-se com a solicitação, da coleta de material germinativo sêmen - pela esposa ou pelos familiares. Tal situação inusitada tem despertado o risco de conflitos éticos importantes para o profissional médico, a esposa/ requerente e os familiares, os direitos da criança a ser gerada e colocando em questão a coleta do material post mortem e sua utilização.

Este trabalho justifica-se pela necessidade de se discutir as implicações legais e éticas da reprodução post mortem no Brasil, suas repercussões sociais com relação à criança a ser gerada e a postura ética do profissional médico envolvido no caso.

\section{OBJETIVO}

Relato de dois casos de pacientes masculinos em morte encefálica, diagnóstico realizado de acordo com a legislação vigente, que foram avaliados pela Organização de Procura de Órgãos do Hospital das Clínicas da Universidade Estadual de Campinas (OPOHC-Unicamp), cujas esposas solicitaram a coleta de sêmen post mortem, com finalidade de inseminação artificial nas solicitantes, com o objetivo de avaliação e repercussão legal da criança a ser gerada, como também, o dilema ético do médico que participa do processo.

\section{CASUÍSTICA E MÉTODO}

Pesquisa de caráter qualitativo e descritivo. Dentre as opções de métodos existentes para a coleta de dados na abordagem qualitativa, optou-se pela pesquisa documental realizada por meio de levantamento bibliográfico médico, ético e legislativo, acerca dos conceitos relativos ao transplante de órgãos, doação de células germinativas - sêmen e óvulos - com finalidade reprodutiva no Brasil e direitos da criança.

\section{Procedimentos de revisão}

Foi realizada pesquisa documental por meio de levantamento bibliográfico, com buscas em plataformas digitais (Google scholar, PubMed, Scielo Brasil, Lilacs), sem qualquer filtro, com data final em maio de 2016, utilizandose as seguintes palavras-chaves: em inglês: brain death and sperm donation; brain death and semen donation; brain death and posthumous semen (or sperm) retrieval; family and donation; donation and autonomy; em português: morte encefálica e doação de órgãos e legislação; morte encefálica e doação de sêmen post mortem; inseminação artificial e coleta de sêmen post mortem; legislação de doação de células germinativas; doação de gametas post mortem; inseminação post mortem e conceito de filiação e linha sucessória; e em espanhol: muerte encefálica y donación de órganos y tejidos; muerte encefálica y donación de células germinativas.

\section{RESULTADOS}

\section{Relatos de casos}

\section{Caso 1}

Homem de 28 anos, casado há 18 meses, sem filhos, vítima de lesão cerebral traumática grave, após um acidente de carro, em 2011. O paciente progrediu para coma não reativo. Um diagnóstico de morte encefálica foi estabelecido, de acordo com o protocolo exigido pelo Conselho Federal de Medicina do Brasil (CFM-Brasil) em sua Resolução 1480/97.7

Após a obtenção do consentimento familiar para a doação dos órgãos, sua esposa expressou desejo de que os médicos coletassem e armazenassem o sêmen do doador para eventual uso próprio, posteriormente, através de técnicas de reprodução assistida.

\section{Caso 2}

Um homem de 31 anos, casado há 24 meses e sem filhos, vítima de lesão cerebral traumática. Após o diagnóstico de morte encefálica, a família autorizou a doação dos órgãos. Após assinar o documento de doação, a esposa solicitou consentimento para a coleta de sêmen para uso final em técnicas de reprodução assistida.

Em nenhum dos dois casos ocorreu a coleta de sêmen, em cumprimento à Resolução $1480 / 97^{7}$ vigente à época dos fatos.

\section{Revisão de Literatura}

Utilizando-se os termos brain death and posthumous retrieval, foram obtidos oito artigos, sendo que quatro deles relacionam-se ao procedimento de coleta $\mathrm{e}$ 
viabilidade do material coletado, que não é o escopo principal do presente estudo.

Tabela 1 - Resultados obtidos em pesquisa em sites

\begin{tabular}{lll}
\hline Autores e publicação & Ano & Assunto \\
\hline
\end{tabular}

Commentary: posthumous harvesting of gametes--a physician's perspective. Soules ${ }^{8} \mathrm{MR}$.

Commentary: legal and ethical aspects of sperm retrieval.

White ${ }^{3} \mathrm{~GB}$

Ethical and legal aspects of sperm retrieval after death or state. Strong $\mathrm{C}^{9}$

\section{Procreation after} death or mental incompetence: medical advance or technology gone awry? 1996

Ohl DA, Park J, Cohen C, Goodman K, Menge $\mathrm{AC}^{10}$ persistent vegetative
Descreve a solicitação de coleta de irmã de paciente em ME, e discute o destino do material e sua responsabilidade.

Descreve sobre a situação do "consentimento razoavelmente inferido", sendo uma justificativa para a recuperação post mortem pode abrir uma situação fora do interesse dos sobreviventes ou da pessoa a ser gerada.

Refere que a coleta somente deve ser realizada como consentimento prévio a morte.

Relato de caso de pacientes em ME ou lesão de sistema nervoso central grave. Descrevem procedimento de retirada e implicações legais do ato. Questionam desejo do paciente e a criança a ser gerada.
Em avaliação da legislação brasileira sobre o tema, ao procurarmos no sítio eletrônico do Conselho Federal de Medicina (CFM) observamos que este iniciou a regulamentação da atividade médica nos casos de reprodução humana assistida em 1992, com atualizações constantes, mantendo a postura de que a reprodução post mortem será permitida com o consentimento informado em vida, e sempre com a coleta realizada em vida, mas não se posiciona com relação à coleta post mortem.

Não foi encontrada nenhuma legislação específica sobre a questão de coletas post mortem.
Tabela 2 - Resoluções do CFM sobre reprodução assistida (RA)

\begin{tabular}{ccc}
\hline $\begin{array}{c}\text { Resoluções do } \\
\text { Conselho Federal de } \\
\text { Medicina(CFM }\end{array}$ & $\begin{array}{c}\text { Ano de } \\
\text { Publicação }\end{array}$ & Assunto \\
\hline
\end{tabular}

Resolução CFM

1.358/92 D.O.U., 19/11/92 Seção I, p.16053 $3^{11}$

1992

Resolução CFM no 1.957/2010 D.O.U. de 06 de janeiro de

Resolução CFM no 2.013, DE 16 DE Abril de 2013. D.O.U. 09 maio de 2013, Seção I, p.119-120.13

Resolução CFM no $2.121 / 2015$. Publicada no D.O.U. de 24/09/2015, Seção I, p. $117^{14}$ 2011, Seção I, p.79 ${ }^{12}$
Consentimento informado: obrigatório e extensivo aos pacientes inférteis e doadores. Conflitos éticos: observa-se: a necessidade de vinculo matrimonial. As informações devem também atingir dados de caráter biológico, jurídico, ético e econômico. Documento de consentimento informado será em formulário especial, e estará completo com a concordância, por escrito, da paciente ou do casal infértil.

Consentimento informado pacientes submetidos às técnicas de reprodução assistida, inclusive aos doadores. Não constitui ilícito ético a reprodução humana assistida post mortem desde que haja autorização prévia específica da(o) falecida(o) para uso do material biológico crio preservado de acordo com a legislação vigente.

VIII - Reprodução Humana Assistida post mortem. É possível desde que haja autorização prévia específica do(a) falecido(a) para o uso do material biológico crio preservado, de acordo com a legislação vigente.

VIII - Reprodução Assistida Post mortem - É permitida a reprodução assistida postmortem desde que haja autorização prévia específica do(a) falecido(a) para o uso do material biológico crio preservado, de acordo com a legislação vigente. - obrigatório a todos os

\section{DISCUSSÃO}

No presente trabalho, trataremos da discussão de coleta de material germinativo post mortem, de acordo com a visão dos direitos da criança a ser gerada e os conflitos existentes entre a ética e o direito na ação médica. 


\section{Direito da Criança}

O Código Civil Brasileiro de 2002, ${ }^{15}$ que regulamenta os conceitos de família e sociedade, especifica e reconhece a paternidade em seu artigo 1597: "I - nascidos 180 (cento e oitenta) dias, pelo menos, depois de estabelecida a convivência conjugal; II - nascidos nos 300 (trezentos) dias subsequentes à dissolução da sociedade conjugal, por morte, separação judicial, nulidade e anulação do casamento; as quais têm por finalidade fixar o momento da concepção, definindo a filiação e certificando a paternidade, imputando-Ihe os direitos e deveres; III havidos por fecundação artificial homóloga, mesmo que falecido o marido", também são presumidos como concebidos "na constância do casamento", o que faz com que a discussão deixe de centrar-se na questão da filiação para recair sobre a questão do consentimento e da decisão sobre o direito de nascer. $\mathrm{Na}$ avaliação de linha sucessória, poderia ocorrer divergência com relação à paternidade e direitos, uma vez que a viúva geraria um filho de pessoa falecida: caso tivesse o consentimento informado, o direito seria imediato.

Frente à situação da criança e sendo obrigação da sociedade protegê-la, no Brasil, o CFM ${ }^{12}$ deixa claro, desde 2010, a necessidade do consentimento informado, inclusive para a preservação do material e posterior utilização, não deixando dúvidas que a decisão será tomada em vida. Conceituar que a criança criada em lar monoparental teria prejuízo em sua formação é um argumento que não se sustenta, uma vez que a própria Constituição Federal ${ }^{16}$ define esse como um dos modelos legais de família, entendimento compartilhado pela Convenção Internacional dos Direitos da Criança, ${ }^{17}$ assinada em 1989, na Assembleia Geral das Nações Unidas, da qual o Brasil é signatário.

O que se vê na estrutura jurídica brasileira é a ausência de normas específicas que garantam o direito da criança gerada em inseminação post mortem. Não há previsão suficiente de normas em nosso ordenamento jurídico e nem mesmo a própria sociedade posicionou-se a respeito desse tema. Ao ser tratada como filho, a criança tem atendidas necessidades como alimentação, moradia e educação, e é considerada como filho nas relações sociais, sendo reconhecida como tal pela sociedade e pela família com a qual convive. A formação do filho como ser humano é o tratamento de maior valor e que lhe traz o máximo de benefícios, mas isso nem a lei e nem o sangue lhe garantem. Talvez a verdadeira filiação esteja na dedicação do cuidar do que na procedência do sêmen, compactuando com o que é mencionado no artigo 227 da Constituição Federal. ${ }^{16}$

A Constituição Federal 16 em seu artigo $1^{\circ}$, inciso III, passou a adotar o princípio da dignidade da pessoa humana, de maneira que a pessoa surge como valor e possui dignidade pela sua própria existência. No Código Civil de $2002{ }^{15}$ e na Constituição Federal de $1988,{ }^{16}$ a família é admitida quando há o reconhecimento da união estável e, no caso se ser família monoparental, quando é formada por um dos pais e seus descendentes, também possui o mesmo entendimento.

Tal descrição é responsável pela quebra do monopólio do casamento como legitimador da família e pela abertura de um conceito de família baseada na boa relação e na valorização humana, em lugar daquele que a definia a partir de uma estrutura sacra, criada pela igreja.

Neste sentido, podemos citar Barroso e Martel, ${ }^{18}$ ao afirmarem que "um indivíduo não tem poder sobre o início da própria vida. Sua concepção e seu nascimento são frutos da vontade alheia".

Esse, portanto, seria mais um dilema ético que podemos definir como "uma escolha da qual não participamos".

\section{Aspectos Éticos}

\section{O Papel do Médico}

O médico deve pautar sua conduta ética, de acordo com seu Código Deontológico. No Brasil, o Código de Ética Médica (CEM) ${ }^{19}$ elaborado e publicado pelo CFM-Brasil em 2009, possui um capítulo específico sobre a doação de órgãos. Com relação à doação de órgãos, tanto a lei 9434/976, quanto a Resolução 1480/977, deixam claro em seus artigos que tal legislação não se aplica a gametas.

As situações descritas nos casos da literatura e enfrentadas pelos autores dão-se em momento de morte súbita, inesperada, em que a mistura de sentimentos dos familiares pode estar em evidência, tais como a efetivação da perda e os desejos, talvez, de uma "imortalidade" do falecido.

Soules et al (1999) ${ }^{8}$ descreveram a solicitação de retirada de ovários para posterior coleta de óvulos e sua preservação. A solicitante, no caso, era a irmã da falecida. Como deveriam os médicos lidar com tal situação? Quem seria autorizado a solicitar e utilizar o material? O momento agudo de dor e perda seria o contexto para se realizar essa solicitação? O médico deve obrigatoriamente solicitar o consentimento, caso ele exista? Ou mesmo o consentimento presumido das pessoas envolvidas? O médico seria negligente se não fornecesse aconselhamento adequado para a família nesse momento?

Bahm et al ${ }^{20}$ propõem uma política ética para esses casos, sugerindo que, diante da solicitação da esposa/ 
companheira, existiria a necessidade de espera para utilização do material, por pelo menos 12 meses, além de acompanhamento psicológico para suporte na decisão a ser tomada, sendo essa talvez a melhor saída frente à total ausência de legislação específica.

Devido à ausência de jurisdição específica, o médico, na maioria das vezes, toma a decisão sozinho, criando conflitos com os familiares, a instituição e, ainda pior, criando conflito interno por não saber se fez a coisa certa.

\section{CONCLUSÃO}

O presente estudo revela que a ausência de legislação específica sobre o tema dificulta a decisão segura pelas partes envolvidas. A criança a ser gerada não possui direitos bem estabelecidos em nosso arcabouço jurídico, não se modernizando com relação aos desafios de novas opções de reprodução humana. Ao médico cabe cumprir a orientação ética, esclarecendo à família a situação a ser criada e suas repercussões sociais.

\section{ABSTRACT}

Recent data shows that $43 \%$ of the families in Brasil still do not autorize organ donation. Therefore understanding which variables play a role at this moment and the complex decision-making process on organ donation is necessary so that new efforts seeking to raise families consent in the country can be implemented. This present work aims: a) to introduce behavioral concepts with the potential to enrich our understanding about the decision making process on organ donation like framing, status quo, social norms, inertia and procrastination; b) to bring forward experiments and public policies already applied worldwide inspired in this behavioral concepts and its results. This reflexive study is based on the Behavioral Economics findings. We conclude from this current reflection that these behavioral concepts present relevant explanatory potential. To incorporate them in the decision-making process model indicates not only a possible contribution to families approach, but also to the construction of campaigns and in the future for a better choice architecture design (nudge).

Keywords: Organ Donation; Behavioral Economics; Decision Making.

\section{REFERÊNCIAS}

1. C.M. Rothman, C.M. A method for obtaining viable sperm in the postmortem state, Fertil. Steril. 1980 Nov;34(5):512 <https://www. ncbi.nlm.nih.gov/pubmed/7439417> (acessado em 12 de novembro de 2014).

2. G. Bahadur. Death and Conception, Human Reproduction, 2002 Oct;17(10):2769-75, <https://academic.oup.com/humrep/ article/17/10/2769/607780> (acessado 14 de fevereiro de 2015).

3. White, G.B., 1999. Commentary: legal and ethical aspects of sperm retrieval, J Law Med Ethics. 1999 Winter;27(4);295:359-61, , <https:// www.ncbi.nlm.nih.gov/pubmed/11067618> (acessado 15 de fevereiro de 2015).

4. Landau, R., 2004. Posthumous sperm retrieval for the purpose of later insemination or IVF in Israel: an ethical and psychosocial critique. Hum. Reprod. 2004 Sep;19(9):1952-6. DOI: 10.1093/humrep/deh360.

5. K. Tremellen, J. Savulescu, A discussion supporting presumed consent for posthumous sperm procurement and conception, Reproductive BioMedicina Online 2015 Jan;30(1):6-13. <http://www.rbmojournal. com/article/S1472-6483(14)00542-2/pdf> (acessado 3 de janeiro de 2016)
6. Lei no 9.434, de 4 de fevereiro de 1997. Dispõe sobre a remoção de órgãos, tecidos e partes do corpo humano para fins de transplante e tratamento e dá outras providências. Publicado D.O.U. 05/02/1997 página 2191. (1997) < http://legislacao.planalto.gov.br/legisla/ legislacao.nsf/Viw_Identificacao/lei\%209.434-1997?OpenDocum ent\&TSPD_101_R0=c4ca48a385d9b55ee576995149387a21nS00 000000000000000712 eaeb3ffff000000000000000000000000 00005b660f4100385028d908282a9212ab20000f603533e61bc07 8a74124438381444882b803120123b70b3e0acac925134

7. CFM Resolução no 1.480/97, Publicada no D.O.U. de 2108.97. página 18.227 (1997), <http://www.portalmedico.org.br/ resolucoes/cfm/1997/1480_1997.htm> (acessado 10 de fevereiro de 2014).

8. Soules MR. Commentary: posthumous harvesting of gametes-a physicians perspective. J Law Med Ethics. 1999 Winter;27(4);362-5:295.

9. Strong $C$. Ethical and legal aspects of sperm retrieval after death or persistent vegetative state. J Law Med Ethics. 1999 Winter;27(4);295:347-58 
A coleta de sêmen post mortem para reprodução humana assistida e os direitos da criança

10. Ohl DA, Park J, Cohen C, Goodman K, Menge AC. Procreation after death or mental incompetence: medical advance or technology gone awry? Fertil Steril. 1996 Dec;66(6):889-95.

11.CFM. Resolução1.358/92.D.O.U.,19 denovembrode1992Seçãol, p.1603. http://www.portalmedico.org.br/resolucoes/cfm/1992/1358_1992.htm. (acessado 10 de novembro de 2015).

12. CFM Resolução 1.957/10. D.O.U. 06 de janeiro de 2010. Seção I,p.79. http://www.portalmedico.org.br/resolucoes/cfm/2010/1957_2010.htm. (acessado em 10 de novembro de 2015).

13. CFM Resolução no 2.013. D.O.U. 09 maio de 2013, Seção I, p.119-120. https://www.cremesp.org.br/?siteAcao=LegislacaoBusca\&nota=712. (acessado 10 de novembro de 2015).
14. CFM Resolução n 2.121/15. D.O.U. 24 de setembro de 2015, Seção I, p. 117. http://www.portalmedico.org.br/resolucoes/cfm/2015/2121_2015. pdf. (acessado em 10 de novembro de 2015).

15. Lei 10.406 de 2002. Institui o Código Civil Brasileiro. D.O.U. de 11 de janeiro de 2002. p.1 http://legislacao.planalto.gov.br/legisla/legislacao. nsf/Viw_Identificacao/lei\%2010.4062002?OpenDocument\&TSPD_101 R0=5abc5cc9f34f95f2c0f85af312e99ae8nM70000000000000000 $\mathrm{d} 13 \overline{\mathrm{d}}$ bcb1ffff00000000000000000000000000005bcf8c04008a41d6430828 2a9212ab20008dca76fb6887249ab 